\title{
Alpha correlations in terms of the hyper-Pfaffian
}

\author{
Takahiro Mizusaki ${ }^{1, *}$ and Peter Schuck $^{2,3}$ \\ ${ }^{1}$ Institute of Natural Sciences, Senshu University, Tokyo 101-8425, Japan \\ ${ }^{2}$ Institut de Physique Nucléaire, IN2P3-CNRS, Université Paris-Sud, 91406 Orsay, France \\ ${ }^{3}$ Univ. Grenoble Alpes, CNRS, LPMMC, 38000 Grenoble, France
}

\begin{abstract}
We find that the overlap between alpha-like four-body condensation wave function and $m$-scheme basis state can be represented by the hyper-Pfaffian, which is a natural extension of the Pfaffian for the paircondensation wave function. This overlap is useful for nuclear structure calculations with the variational Monte Carlo method. As the hyper-Pfaffian has, however, only a few mathematical relations, it is quite difficult to use for numerical computations. We also find that a specific case of hyper-Pfaffian can be expressed by the sum of standard Pfaffians.
\end{abstract}

\section{Introduction}

The alpha states for $\mathrm{C}$ and $\mathrm{O}$ have been studied over a halfcentury. On the other hand, alpha-like correlation or quartet structure in the shell model calculations has also been discussed, for example, Ref. [1]. The alpha-like fournucleon correlations have been discussed in Refs. [2,3] with the closure approximation. Recently renewed interests have been paid to such quartet structures in the nuclear shell model by N. Sandulescu and his collaborators [4-6] with the use of recursion relations.

Recently the variational Monte Carlo (VMC) method with the pair-condensate trial state has been applied to fermion many-body systems [7, 8], including the nuclear shell model $[9,10]$. The VMC trial wave function with the pair-condensation state is given as

$$
\left|\Psi^{2 n}\right\rangle=\left(\frac{1}{2} \sum f_{i j} c_{i}^{\dagger} c_{j}^{\dagger}\right)^{n}|0\rangle
$$

where $c^{\dagger}$ 's are nucleon creation operators, $n$ is the number of pairs and $|0\rangle$ is the fermion vacuum. The overlap between this pair-condensate wave function and the $m$ scheme basis $|\boldsymbol{m}\rangle$, which is defined as $|\boldsymbol{m}\rangle=c_{m_{1}}^{\dagger} \cdots c_{m_{2 n}}^{\dagger}|0\rangle$, is shown by

$$
\left\langle\boldsymbol{m} \mid \Psi^{2 n}\right\rangle=n ! P f(\tilde{f})
$$

where $P f$ is the Pfaffian. (For its detail, see $[9,10]$ for example.) Note that the Pfaffian has been recently studied in the overlap between the HFB wave functions, for examples, [11-13].

The energy by the VMC is formulated as

$$
E=\sum_{m} \rho(m) E_{L}(m),
$$

where the sum regarding $m$ is estimated by the Monte Carlo sampling. The local energy $E_{L}(m)$ is defined as

$$
E_{L}(m)=\sum_{m^{\prime}} h_{m, m^{\prime}} \frac{\left\langle m^{\prime} \mid \phi\right\rangle}{\langle m \mid \phi\rangle} .
$$

The Monte Carlo sampling is carried out with the probability density $\rho(m)$, defined by

$$
\rho(m)=\frac{|\langle m \mid \phi\rangle|^{2}}{\sum_{m}|\langle m \mid \phi\rangle|^{2}},
$$

where $\rho(m) \geq 0$ and $\sum_{m} \rho(m)=1$. Thus, what we need to evaluate the energy, is the overlaps $\langle m \mid \phi\rangle$. If we obtain the gradient vector regarding the parameters contained in the trial state, we can perform the energy minimization with the Monte Carlo method. In this formulation, the complicated matrix element of the Hamiltonian between the two trial states is not explicitly needed. Therefore we focus on the overlap matrix element hereafter.

\section{Quartet-condensate wave function and hyper-Pfaffian}

In this work, we extend the pair-condensate wave function to the quartet-condensate wave function as

$$
\left|\Psi^{4 n}\right\rangle=\left(\frac{1}{4 !} \sum f_{p q r s} c_{p}^{\dagger} c_{q}^{\dagger} c_{r}^{\dagger} c_{s}^{\dagger}\right)^{n}|0\rangle
$$

where $n$ is the number of quartets. We found [14] that the overlap between this quartet-condensate wave function and $m$-scheme basis, $|\boldsymbol{m}\rangle=c_{m_{1}}^{\dagger} \cdots c_{m_{4 n}}^{\dagger}|0\rangle$, can be expressed by the hyper-Pfaffian as

$$
\left\langle\boldsymbol{m} \mid \Psi^{4 n}\right\rangle=\left\langle\boldsymbol{m}\left|\left(\sum_{p<q<r<s} f_{p q r s} c_{p}^{\dagger} c_{q}^{\dagger} c_{r}^{\dagger} c_{s}^{\dagger}\right)^{n}\right| 0\right\rangle=n ! P f^{[4]}(f)
$$

\footnotetext{
*e-mail: mizusaki@isc.senshu-u.ac.jp
} 
where the $P f^{[k]}$ is the hyper-Pfaffian by Jean-Gabriel Luque and Jean-Yves Thibon [15]. It is defined for fully anti-symmetric tensor $M_{i_{1}, \cdots, i_{k}}\left(M_{i_{\sigma}(1), \cdots, i_{\sigma}(k)}=\right.$ $\operatorname{sgn}(\sigma) M_{i_{1}, \cdots, i_{k}}$ for any permutation $\left.\sigma\right)$ as,

$$
P f^{[k]}(M) \equiv \sum_{\sigma \in \mathbb{G}_{k n, k}} \operatorname{sgn}(\sigma) \prod_{i=1}^{n} M_{\sigma(k(i-1)+1), \sigma(k(i-1)+2), \cdots, \sigma(k i)}
$$

where permutation $\sigma$ is restricted with the following condition as,

$$
\begin{aligned}
\mathfrak{S}_{k n, k} \equiv & \left\{\sigma \in \mathfrak{S}_{k n} \mid \sigma(k(i-1)+1)<\cdots<\sigma(k i)\right), \\
& \sigma(k(p-1)+1)<\sigma(k p+1)) \\
& 1 \leq i \leq n, 1 \leq p \leq n-1\} .
\end{aligned}
$$

In the Appendix, we show two examples. The hyperPfaffian has been very recently studied by several mathematicians [15-18]. Eq.(7) with this hyper-Pfaffian is a natural extension of the usual Pfaffian form. In general $k$-body condensation corresponds to the $k$-th order hyperPfaffian. However, the hyper-Pfaffian has, unfortunately, only a few mathematical relations, unlike the Pfaffian.

Next, we try to find useful relations by assuming the following form as

$$
\left\langle\boldsymbol{m} \mid \Psi^{4 n}\right\rangle=\left\langle\boldsymbol{m}^{\pi} \boldsymbol{m}^{v}\left|\left(\frac{1}{4} \sum f_{p q r s} c_{p}^{\pi \dagger} c_{q}^{\pi \dagger} c_{r}^{\nu^{\dagger}} c_{s}^{v^{\dagger}}\right)^{n}\right| 0\right\rangle
$$

where $\pi$ and $v$ are proton and neutron labels. The $m$ scheme state is also a product of the proton and neutron $m$-scheme states $\left|\boldsymbol{m}^{\pi}\right\rangle$ and $\left|\boldsymbol{m}^{v}\right\rangle$, respectively. This form means alpha-like condensation.

For $n=2$ and 3, we easily find that the corresponding hyper-Pfaffians can be written with the sum of standard Pfaffians. For $k=4, n=2$, the hyper-Pfaffian is reduced into the ordinary Pfaffian as

$$
P f^{[4]}(f)=\sum_{\sigma \in \mathbb{E}_{4}} \operatorname{sgn}(\sigma) P f\left(f_{\sigma(1) \sigma(2), \sigma(3) \sigma(4)}\right)
$$

where $\sigma(1)<\sigma(2)$ and $\sigma(3)<\sigma(4)$. For $k=4, n=3$, the hyper-Pfaffian is also reduced into the ordinary Pfaffian as

$$
\begin{aligned}
P f^{[4]}(f) & =\sum_{\sigma \in \mathfrak{S}_{6}} \operatorname{sgn}(\sigma)\left[\operatorname{Pf}\left(f_{\sigma(1), \sigma(2), \sigma(3), \sigma(4), \sigma(5), \sigma(6)}^{3}\right)\right. \\
& \left.+z_{\sigma(1), \sigma(2), 9,10} \operatorname{Pf}\left(f_{\sigma(3), \sigma(4), \sigma(5), \sigma(6)}^{2}\right)\right]
\end{aligned}
$$

where $\sigma(1)<\sigma(2), \sigma(3)<\sigma(4)$ and $\sigma(5)<\sigma(6)$. These relations are derived in [14], while for $n>3$, there is no simple relation. To find a more general formula beyond this limitation and the gradient formula is currently under our investigation.

This work was supported by the research grant of the Senshu Research Abroad Program (2018) for one of the authors (T.M.).

\section{Appendix: Example of the hyper-Pfaffian}

Here we show two examples of the hyper-Pfaffian. For the $k=4, n=1$,

$$
P f^{[4]}[f]=f_{1234},
$$

For $k=4, n=2$,

$$
\begin{aligned}
P f^{[4]}(f) & =f_{1234} f_{5678}-f_{1235} f_{4678}+f_{1236} f_{4578} \\
& -f_{1237} f_{4568}+f_{1238} f_{4567}+f_{1245} f_{3678} \\
& -f_{1246} f_{3578}+f_{1247} f_{3568}-f_{1248} f_{3567} \\
& +f_{1256} f_{3478}-f_{1257} f_{3468}+f_{1258} f_{3467} \\
& +f_{1267} f_{3458}-f_{1268} f_{3457}+f_{1278} f_{3456} \\
& -f_{1345} f_{2678}+f_{1346} f_{2578}-f_{1347} f_{2568} \\
& +f_{1348} f_{2567}-f_{1356} f_{2478}+f_{1357} f_{2468} \\
& -f_{1358} f_{2467}-f_{1367} f_{2458}+f_{1368} f_{2457} \\
& -f_{1378} f_{2456}+f_{1456} f_{2378}-f_{1457} f_{2368} \\
& +f_{1458} f_{2367}+f_{1467} f_{2358}-f_{1468} f_{2357} \\
& +f_{1478} f_{2356}-f_{1567} f_{2348}+f_{1568} f_{2347} \\
& -f_{1578} f_{2346}+f_{1678} f_{2345} .
\end{aligned}
$$

As $n$ increases, the number of terms explosively increases.

\section{References}

[1] A. Arima and V. Gillet, Annal of Phys. 66, 117 (1971).

[2] M. Hasegawa, S. Tazaki, R. Okamoto, Nucl. Phys. A592 45 (1995).

[3] M. Hasegawa, S. Tazaki, Nucl. Phys. A633 266 (1998).

[4] N. Sandulescu and D. Negrea, J. Dukelsky, and C. W. Johnson, Phys. Rev. C 85 061303(R) (2012).

[5] N. Sandulescu, D. Negrea, and C. W. Johnson, Phys. Rev. C86, 041302(R) (2012).

[6] M. Sambataro and N. Sandulescu, Phys. Rev. C93, 054320 (2016).

[7] M. Bajdich, L. Mitas, G. Drobny, L. K. Wagner, and K. E. Schmidt, Phys. Rev. Lett. 96, 130201 (2006).

[8] D. Tahara and M. Imada, J. Phys. Soc. Jpn. 77, 114701 (2008).

[9] T. Mizusaki and N. Shimizu, Phys. Rev. C85, 021301(R) (2012).

[10] N. Shimizu and T. Mizusaki, Phys. Rev. C98, 054309 (2018).

[11] L. M. Robledo, Phys. Rev. C79, 021302(R) (2009).

[12] B. Avez and M. Bender, Phys. Rev. C85, 034325 (2012).

[13] T. Mizusaki and M. Oi, Phys. Lett. B715, 219 (2012).

[14] T. Mizusaki and P. Schuck, Bulletin of the Institute of Natural Sciences, Senshu University 50, 19, (2019).

[15] J.-G. Luque, J.-Y. Thibon, Adv. in Appl. Math. 29 620 (2002).

[16] A.I. Barvinok, Mathematical Programming, 69449 (1995).

[17] D. Redelmeier, Ph.D. University of Waterloo (2006).

[18] Sho Matsumoto, Journal of Algebra 320612 (2008). 